\title{
On projective group properties of the $6 D$ pseudo-Riemannian space
}

\author{
Zolfira Zakirova* \\ Kazan State Energy University, Kazan, Russia
}

\begin{abstract}
We study the six-dimensional pseudo-Riemannian spaces with two time-like coordinates that admit non-homothetic infinitesimal projective transformations. The metrics are manifestly obtained and the projective group properties are determined. We also find a generic defining of projective motion in the 6 -dimensional rigid $h$-space.
\end{abstract}

\section{Introduction}

The problem of defining $2 D$ Riemannian manifolds which admit projective motions, i. e. continuous transformation groups preserving geodesics dates back to S.Lie who considered it in [1]. ${ }^{1}$ In more recent times, that was A.V.Aminova who has got a complete solution to this problem [3]. For the Riemannian manifolds of dimension greater than 2 the same problem has been solved by G.Fubini [4] and A.S.Solodovnikov [5].

Note that they essentially used in their studies positive definiteness of metrics under consideration. Without this positivity condition, considering pseudo-Riemannian spaces, the problem is much more complicated and requires absolutely new method of solution.

In later paper, 6] A.V.Aminova has classified all the Lorentzian manifolds of dimension $\geq 3$ that admit nonhomothetic projective or affine infinitesimal transformations. In each case, there were determined the corresponding maximal projective and affine Lie algebras.

The General problem is to classify the n-dimensional pseudo-Riemannian spaces admitting projective motions, i. e. continuous transformation groups preserving geodesics.

This problem is not solved yet for pseudo-Riemannian spaces with arbitrary signature.

Our concrete problem here is to study a projective group properties of a 6-dimensional pseudoRiemannian space with signature $\left[++--_{-}\right]$, which admits projective motions, i. e. continuous transformation groups preserving geodesics.

The Method we use here is the method of the skew-normal frames and general approach to investigation of projective motions of pseudo-Riemannian manifolds due to A. V. Aminova.

\section{The metrics of the rigid $h$-spaces}

Let me remind that vector field $X$ on a pseudo-Riemannian manifold $(M, g)$ is an infinitesimal projective transformation if and only if [7]

$$
\begin{gathered}
L_{X} g=h, \quad \text { generalized Killing equation } \\
\nabla h(Y, Z, W)=2 g(Y, Z) W \varphi+g(Y, W) Z \varphi+g(Z, W) Y \varphi
\end{gathered}
$$

\footnotetext{
*E-mail: zolya@itep.ru
}

${ }^{1}$ Another important result was obtained by A.Z.Petrov [2], who classified geodesically equivalent pseudo-Riemannian spaces $V^{3}$. 
where $(Y, Z, W) \in T(M), \varphi=\frac{1}{n+1} \operatorname{div} X$.

In order to find a pseudo-Riemannian space admitting a nonhomothetic infinitesimal projective transformation, one needs to integrate the Eisenhart equation.

Let us introduce the following definitions.

Definition 1: Pseudo-Riemannian manifolds for which there exist nontrivial solutions $h \neq c g$ to the Eisenhart equation are called $h$-spaces.

The problem of determining such spaces depends on the type of $h$-space, i. e. on the type of the bilinear form $L_{X} g$ determined by the characteristic of the $\lambda$-matrix $(h-\lambda g)$. If the Segre characteristics of the tensor $L_{X} g$ is $[a b c \ldots]$, we call the corresponding $h$-space the space of $[a b c \ldots]$ type. The number of possible types depends on the dimension and the signature of the pseudo-Riemannian space. We restrict ourselves to considering the rigid $h$-spaces.

Definition 2: $h$-spaces with distinct bases of prime divisors of the $\lambda$-matrix are called rigid $h$-spaces.

So, we investigate the rigid $h$-spaces, i. e. $h$-spaces of the [111111], [21111], [2211], [3111], [321], [33], [411] and [51] types.

Using the technics integrating in skew-normal frames, developed by A.V.Aminova, one finds the metrics of these $h$-spaces.

In particular, the metric of the $h$-space of [2211] type is

$$
\begin{gathered}
g_{i j} d x^{i} d x^{j}=e_{2}\left(f_{4}-f_{2}\right)^{2} \Pi_{\sigma}\left(f_{\sigma}-f_{2}\right)\left\{2 A d x^{1} d x^{2}-A^{2} \Sigma_{1}\left(d x^{2}\right)^{2}\right\}+ \\
e_{4}\left(f_{2}-f_{4}\right)^{2} \Pi_{\sigma}\left(f_{\sigma}-f_{4}\right)\left\{2 \tilde{A} d x^{3} d x^{4}-\tilde{A}^{2} \Sigma_{2}\left(d x^{4}\right)^{2}\right\}+\sum_{\sigma} e_{\sigma} \Pi_{i}^{\prime}\left(f_{i}-f_{\sigma}\right)\left(d x^{\sigma}\right)^{2}
\end{gathered}
$$

where

$$
\begin{array}{cl}
A=\epsilon x^{1}+\theta\left(x^{2}\right), & \tilde{A}=\tilde{\epsilon} x^{3}+\omega\left(x^{4}\right), \\
\Sigma_{1}=2\left(f_{4}-f_{2}\right)^{-1}+\sum_{\sigma}\left(f_{\sigma}-f_{2}\right)^{-1}, & \Sigma_{2}=2\left(f_{2}-f_{4}\right)^{-1}+\sum_{\sigma}\left(f_{\sigma}-f_{4}\right)^{-1},
\end{array}
$$

$f_{2}=\epsilon x^{2}, f_{4}=\tilde{\epsilon} x^{4}+a, \epsilon, \tilde{\epsilon}=0,1, a$ is a constant which is nonzero when $\tilde{\epsilon}=0, f_{\sigma}\left(x^{\sigma}\right), \theta\left(x^{2}\right), \omega\left(x^{4}\right)$ are arbitrary functions, $e_{i}= \pm 1, \sigma=5,6$.

The tensor $h_{i j}$ of the $h$-space of [2211] type is

$$
\begin{aligned}
& h_{i j} d x^{i} d x^{j}=2 f_{2} g_{12} d x^{1} d x^{2}+\left(f_{2} g_{22}+A g_{12}\right)\left(d x^{2}\right)^{2}+2 f_{4} g_{34} d x^{3} d x^{4}+ \\
& \left(f_{4} g_{44}+\tilde{A} g_{34}\right)\left(d x^{4}\right)^{2}+\sum_{\sigma} f_{\sigma} g_{\sigma \sigma}\left(d x^{\sigma}\right)^{2}+\left(2 f_{2}+2 f_{4}+\sum_{\sigma} f_{\sigma}+c\right) g_{i j},
\end{aligned}
$$

For every solution $h_{i j}$ of the geodesic Eisenhart equation, there is a quadratic first integral

$$
\left(h_{i j}-4 \varphi g_{i j}\right) \dot{x}^{i} \dot{x}^{j}=\text { const }
$$

where $\dot{x}^{i}$ is the tangent vector to the geodesic.

For the other rigid $h$-spaces we have similar results.

\section{On projective group properties of the 6-dimensional pseudo-Riemannian space}

To move further, we need a necessary and sufficient condition of constant curvature of this $h$-spaces. A necessary and sufficient of condition of constant curvature is expressed by the formula

$$
R_{j k l}^{i}=K\left(\delta_{k}^{i} g_{j l}-\delta_{l}^{i} g_{j k}\right), \quad K=\text { const. }
$$


Calculating components of the curvature tensor of the rigid $h$-spaces and substituting into this equality, one obtains a necessary and sufficient conditions of constant curvature of this $h$-space.

In particular, for the $h$-space of [2211] type

$$
\rho_{p}-\rho_{\sigma p}=\rho_{p}-\rho_{p q}=\epsilon=\tilde{\epsilon}=0 \quad(p \neq q, p, q=2,4, \sigma=5,6),
$$

where

$$
\begin{gathered}
\rho_{p}=-\frac{1}{4} \sum_{\sigma} \frac{\left(f_{\sigma}^{\prime}\right)^{2}}{\left(f_{\sigma}-f_{p}\right)^{2} g_{\sigma \sigma}}, \quad \rho_{p q}=-\frac{1}{4} \sum_{\sigma} \frac{\left(f_{\sigma}^{\prime}\right)^{2}}{\left(f_{\sigma}-f_{p}\right)\left(f_{\sigma}-f_{q}\right) g_{\sigma \sigma}}, \\
\rho_{\sigma p}=-\frac{1}{4} \frac{\left(f_{\sigma}^{\prime}\right)^{2}}{\left(f_{\sigma}-f_{p}\right) g_{\sigma \sigma}}\left\{\frac{2 f^{\prime \prime}{ }_{\sigma}}{\left(f_{\sigma}^{\prime}\right)^{2}}-\frac{1}{f_{\sigma}-f_{p}}+\sum_{i, i \neq \sigma}\left(f_{i}-f_{\sigma}\right)^{-1}\right\}- \\
\frac{1}{4} \sum_{\gamma, \gamma \neq \sigma} \frac{\left(f_{\gamma}^{\prime}\right)^{2}}{\left(f_{\gamma}-f_{p}\right)\left(f_{\gamma}-f_{\sigma}\right) g_{\gamma \gamma}} .
\end{gathered}
$$

Further, investigating the Eisenhart equations and their integrability conditions for each obtained rigid $h$-spaces we prove some theorems, when give important information about structure projective Lie algebra in the rigid $h$-spaces.

Theorem 1. Any defining function of projective motion in rigid h-spaces of nonconstant curvature can be presented as $\phi=a_{1} \varphi$, where $a_{1}$ is a constant.

Theorem 2. Any covariantly constant symmetric tensor $b_{i j}$ in 6-dimensional rigid h-spaces of nonzero curvature is proportional to the fundamental tensor, $i$. e. $b_{i j}=a_{2} g_{i j}$, where $a_{2}$ is a constant.

From this theorems, one immediately obtains

Theorem 3. The affine group of 6-dimensional rigid h-spaces of non-constant curvature consists of homothetics.

These theorems and linearity of the Eisenhart equation give the general solution of the Eisenhart equation in the rigid $h$-spaces of non-constant curvature in the form

$$
a_{1} h_{i j}+a_{2} g_{i j}
$$

with two arbitrary constants $a_{1}, a_{2}$.

Hence, one obtains

Theorem 4. All projective motions of a 6 -dimensional rigid h-spaces of non-constant curvature are obtained by integrating the equation

$$
L_{\xi} g_{i j}=\xi_{i, j}+\xi_{j, i}=a_{1} h_{i j}+a_{2} g_{i j} .
$$

It leads to the following important group characteristic of rigid $h$-spaces:

Theorem 5. If rigid h-spaces of non-constant curvature admit a nonhomothetic projective Lie algebra $P_{r}$, then this algebra contains the subalgebra $H_{r-1}$ of infinitesimal homothetics of dimension $r-1$.

I am grateful to A.V.Aminova for the constant encouragement and discussions and to C.BurdiK for kind hospitality in Prague and the very nice Conference. The work is partially supported by RFBR grant 04-02-16538-. 


\section{References}

[1] M. G. Konigs: Appl. II to G. Darboux. Lecons sur la theorie generalle des surfaces. IV. (1896), p. 368.

[2] A.Z. Petrov: Uch. zap. Kazan. un-ta. (1949), Vol. 109, no. 3, p. 7 (in Russian).

[3] A. V. Aminova: Iz. VUZ 30 (1990), no. 6, p. 1 (in Russian).

[4] G. Fubini: Mem. Acc. Torino. Cl. Fif. Mat. Nat. (1903) Vol. 53, N2, p. 261.

[5] A. S. Solodovnikov: Uspekhi Mat. Nauk (1956), no. 11, p. 45 (in Russian).

[6] A. V. Aminova: Uspekhi Mat. Nauk 50 (1995), no. 1(301), p. 69 (in Russian).

[7] L. P. Eisenhart: Riemannian geometry M.:IL (1948) (in Russian). 